\title{
Flow induced Energy Losses in the Exhaust Port of an Internal Combustion Engine
}

\author{
Yue Wang \\ Bernhard Semlitsch* \\ $\mathrm{PhD}$. \\ Graduate Student \\ Royal Institute of Technology (KTH) \\ Stockholm, Sweden \\ Mihai Mihaescu \\ Docent, Member of ASME \\ Laszlo Fuchs \\ Professor, Member of ASME \\ CCGEx, Linné Flow Center, Department of Mechanics \\ Royal Institute of Technology (KTH) \\ Stockholm, Sweden
}

CCGEx, Department of Mechanics Linné Flow Center, Department of Mechanics

Royal Institute of Technology (KTH)

Stockholm, Sweden

Email: bernhard@mech.kth.se

\begin{abstract}
A numerical study of the flow in the exhaust port geometry of a Scania heavy-duty Diesel engine is performed using the Large Eddy Simulation (LES) and an Unsteady Reynolds Averaged Navier-Stokes (URANS) simulation approach. The calculations are performed at fixed valve positions and stationary boundary conditions to mimic the setup of an air flow bench experiment, which is commonly used to acquire input data for one-dimensional engine simulations. The numerical results are validated against available experimental data. The complex three-dimensional flow structures generated in the flow field are qualitatively assessed through
\end{abstract}

\footnotetext{
*Address all correspondence for other issues to this author.
} 
visualization and analyzed by statistical means. For low valve lifts, the major source of kinetic energy losses occurs in the proximity of the valve. Flow separation occurs immediately downstream of the valve seat. Strong helical flow structures are observed in the exhaust manifold, which are caused due an interaction of the exhaust port streams in the port geometry.

\section{Nomenclature}

CFD Computational Fluid Dynamics

ICE Internal Combustion Engine

LES Large Eddy Simulation

PIV Particle Image Velocimetry

PSD Power Spectral Density

SGS Sub-grid Scale

URANS Unsteady Reynolds Averaged Navier-Stokes

WSS Wall Shear Stresses

$C_{D} \quad$ discharge coefficient

$d_{e}$ exhaust pipe diameter

$d_{s t} \quad$ valve stem diameter

$A$ area

$K_{C}$ resistance coefficient of contraction

$K_{E}$ resistance coefficient of enlargement

$K_{B} \quad$ loss coefficient of Bend

$p$ pressure

$R \quad$ specific gas constant

$T$ temperature

$V$ velocity

$\gamma$ heat capacity ratio

$\rho$ density

$\tau_{w} \quad$ wall shear stress

\section{Introduction}

Understanding the gas exchange process in an Internal Combustion Engine (ICE) is essential in order to design and construct efficient engines. The reduction of energy losses is one possible way to 
improve the ICE efficiency. In a Diesel engine, approximately 30-40\% of the total available energy remains in the exhaust gases after combustion [1]. A part of this residual energy can be recovered e.g. in a turbocharger, in order to increase the efficiency of the ICE. Hence, the optimal ICE efficiency is accomplished, when the total losses are minimized, while the fuel is expanded as much as possible.

The exhaust port guides the residual gasses from the cylinder to the exhaust manifold, which represents the inlet to the turbocharger turbine. The exhaust system geometry of an ICE engine is spatially restricted to a compact design, which leads to a design of a rather complex piping geometry. This geometrical complexity and the high flow speeds in an ICE induce a significant amount unsteady, complicated, secondary flow motion. As a consequence, a part of the available kinetic energy is lost in the exhaust port, either directly or through formation of flow structures that enhance dissipation.

The efficiency of the turbocharger dependents on the flow structures and the flow profile at the turbine inlet [2]. Therefore, the gas flow in the exhaust port can have a significant impact on the total efficiency of the engine. Further, the gas temperature is an important parameter for the after treatment operability. Caton \& Heywood [3] found that the contributions to heat transfer from large-scale fluid structures indeed dominate over the heat transport in the near wall boundary layer. Hence, the accurate prediction of the flow features in the exhaust port is of relevance and the quality of the exiting flow from the exhaust port has a high impact on the ICE efficiency. However, only a few fundamental systematic studies of the three-dimensional flow in the exhaust system are documented in literature. The complex geometrical constraints challenge the use of experimental measurement techniques for a detailed flow investigation [4]. Therefore, it is essential to predict accurately the flow quantities at the outlet of the exhaust port.

When the entire ICE is simulated for global parametric or optimization studies, a computationally cheap approach is required. In vehicle industry, one-dimensional approaches are widely utilized, due to the rather low computational requirements and the fast available results. However, all the flow scales in the exhaust port cannot be resolved by this formulation. Nonetheless, e.g. variable valve timing optimization is based on one-dimensional simulations of the entire ICE, since the gains depend rather on the global performance than on local details. Variable valve timing can increase the efficiency of the ICE, but also cause an increase of the emissions. Hence, optimization of the variable valve timing is a trade-off of several multi-scale aims. In one dimensional simulation models, as e.g. described in [5], different components of the ICE, e.g. pipes, manifolds, ports, turbine and compressor are represented by a large number of objects like straight pipes, pipe bends, flow splits and junctions. The basic approach is to apply the compressible Navier-Stokes equations for a straight pipe of variable cross section 
assuming only stream-wise (axial) variations in the velocity, density and pressure. Other losses, such as these generated by boundary layers, secondary flow are handled by adding a loss-factor within a given segment. For geometrical complex parts experimental evaluated coefficients are used to account for the losses. Commonly, the effects of complex geometries (including here also the intake and exhaust ports of cylinder heads) on the flow are assessed by using discharge or flow coefficients. Such coefficients are obtained by performing simple experiments with fixed valve lifts at low pressure drops on air flow benches [6]. For such idealized conditions, the pressure differences are much smaller than the gradients occurring in a real engine, the temperatures are lower, and the pulsatile nature of the flow is neglected. Under these restricting assumptions, the provided data is used in one-dimensional computational models. One-dimensional simulations and simple experiments are commonly combined to asses the exhaust port flow [7].

Systematic optimization of the geometry requires the knowledge of the flow field and the sources of the major energy losses. In diverse sections of the exhaust port, the sources of losses are caused due to different generation mechanism. The analyzation of the flow feature details cannot be performed by one-dimensional simulations. For the assessment of flow structure details of the high turbulent flow to optimize geometrical details of the exhaust port geometry, Unsteady Reynolds Averaged Navier-Stokes (URANS) simulations are commonly performed in industry. A turbulence closure model is employed to account for the turbulent flow scales effects and provide numerical solutions of a rather growth numerical grid. Due to ability of handling a smaller computational mesh, the simulation durations are shorter than for more expansive approaches, as e.g. Large Eddy Simulations (LES). This allows several designs to be tested in a reasonable amount of time. However, the turbulence closure models relay on certain assumptions, which restrict the applicability. The accuracy of the URANS simulations relays on the capability of the turbulence closure model to capture all relevant flow phenomena. Thus, the turbulence closure model needs to be verified for each study to be appropriate. A fairly large number of turbulence closure models is available accounting for different flow phenomena. Rather simple models are limited to a wider range of assumptions, where more complex models are computationally more expensive.

The purpose of this paper is to characterize the flow around the valves and in the exhaust port of an automotive Diesel engine at low valve lifts. The complex flow field is investigated for different valve lifts using time resolved LES to enhance the understanding of the involved flow dynamics. The total pressure losses obtained with the numerical simulations are compared to experimental measurements. To be able to compare the numerical simulation results to experimental measurements, the same conditions as in the experiment have been used for the computational simulations. The main focus of this paper is to 
analyze and identify the primary sources of the total pressure losses to enable systematic performance improvement of the geometry. URANS simulations are performed to investigate the implications of the computationally less expansive approach, especially in terms of the total pressure loss estimation.

\section{Geometry and flow case description}

Compressible flow simulations were performed for the exhaust port of the Scania heavy-duty Diesel engine, the D12. The considered geometry consists of five parts, the cylinder, the exhaust port with two pipes conjunct at the downstream end, two valves and an exit pipe as shown in Fig. 1. The exit pipe is 10 exhaust pipe diameters $\left(d_{e}=42 \mathrm{~mm}\right)$ long.

An overview of the computed cases is listed in Tab. 1, where the computational mesh grid size (will be described in the following section), the inlet boundary conditions used, and the corresponding valve lifts are described. The boundary conditions are chosen to match those of the experimental investigation carried out at Scania, where the same exhaust port geometry has been used. At the inlet plane (indicated in Fig. 1 at the bottom of the cylinder), a constant mass flow rate is applied. The static outlet pressure is specified and kept constant at room, ambient conditions in accordance with the experiments. The wall boundaries are modeled as no-slip, adiabatic walls. The sensitivity of the flow solution on the grid resolution is assessed by the cases $\mathrm{C} 1, \mathrm{C} 2$, and $\mathrm{C} 3$ (see Tab. 1) under the same boundary conditions with the valves fixed at $4 \mathrm{~mm}$ valve lift. For the cases $\mathrm{C} 4$ to $\mathrm{C} 6$, the constant mass flow inlet is modified according to the values obtained from the experiments performed at the different valve lifts (i.e. 3 , 4 , and $5 \mathrm{~mm}$ ).

In this paper, the discussed flow field obtained by the numerical simulations are mainly performed using the LES approach. However, URANS formulations with correspondent turbulence closures are commonly used in industry [8]. Therefore, in addition to the LES calculations, URANS simulations were performed for the cases $\mathrm{C} 4, \mathrm{C} 5$, and C6 using the same initial conditions and boundary conditions.

\section{Numerical method}

The governing equations of the compressible flow phenomena are simulated using a finite volume based solver within the framework of the commercial CFD software STAR-CCM+ (by CD-adapco). The discretization schemes used for the LES and URANS simulations were a second order implicit scheme for time integration, while for spatial discretization a bounded central-difference scheme was employed. The bounded central-differencing scheme is essentially based on the normalized variable diagram approach, together with the convection boundedness criterion. Thus, it is a composite normalized variable 
diagram scheme that consists of a blended central differencing and a second-order upwind scheme, or a first order upwind scheme (only when the convection boundedness criterion is violated) [9].

\section{Turbulence modeling}

No explicit modeling of the unclosed Sub-Grid Scale (SGS) term is used for all the LES calculations. This approach assumes that the inherent numerical dissipation represents the effect of the unresolved sub-grid dissipation. The blend of the upwind schemes adds the necessary amount of dissipation to the central-difference scheme. Such LES is usually called implicit LES. It has been shown that second order discretization accuracy is necessary and suitable for the implicit LES approach [10]. Implicit LES has been employed for many different flows within a wide variety of solvers [11]. This approach has been successfully employed in automotive engineering applications and has been validated with Particle Image Velocimetry (PIV) data [12]. A wall function approach has been used, since the grid used has not been designed to resolve all the flow scales at the walls.

For the URANS simulations the standard $k-\varepsilon$ turbulence closure model was used together with classical wall functions [13]. The two-equation turbulence closures (e.g. $k-\varepsilon$ ) may have difficulties in accurately predicting highly anisotropic turbulent flows involving adverse pressure gradients, separation, and secondary motion. However, the model is computationally cheap, and numerically stable. Therefore this approach with the standard $k-\varepsilon$ closure model is widely used in industry.

\section{Computational grids}

The grids are designed with an isotropic hexahedral core mesh and two layers of prism cells at the walls as illustrated in Fig. 2. Additionally, mesh stretching is used towards the outlet region in order to damp possible reflections from the outlet boundary. Three different mesh grid resolutions were used within the LES calculations with an isotropic hexahedral core cell size of $1,0.8$, and $0.6 \mathrm{~mm}$, named grid 1, 2, and 3 (see also Tab. 1), respectively. The total thickness of the two prism layers is half of the hexahedral core cell size and the growth ratio considered between the layers was 1.2. The influence of the grid resolution on the computed solution is assessed by a grid sensitivity study over the three different grids, i.e. grid 1, 2, and 3. These are corresponding to the cases C1, C2, and C3 in Tab. 1, respectively. The total pressure loss, over the inlet to the outlet of the exit pipe, is the essential parameter assessed. The computed values of $5.9 \mathrm{kPa}$ for case $\mathrm{C} 1,5.7 \mathrm{kPa}$ for case $\mathrm{C} 2$, and $5.6 \mathrm{kPa}$ for case $\mathrm{C} 3$ show a monotonically convergence to a lower bound.

A comparison of the time-averaged velocity magnitude and the turbulence kinetic energy profiles, 
obtained with the three different grids, is shown in Fig. 3 and Fig.4, respectively. The locations chosen are a horizontal line in the near valve gap region (shown in Fig. 2), which has not the same extend on both sides of the valve stem, and the vertical and the horizontal line at $y=0 d_{e}$ (see Fig. 1). The time-averaged velocity magnitude profiles in the near valve gap region exhibit slight differences, where the velocity magnitude peak is slightly (about two mesh cells) shifted for the different grids. This is induced due to the rather sensitive event of flow separation from the blunt surfaces at the valve seat and valve head, which is partially triggered by the developing flow recirculation region. (A time instant of the velocity magnitude shown in Fig.7, illustrates the highly unsteady flow motion, which causes the difference in the velocity magnitude profiles in the near valve region.) The time-averaged velocity magnitude profiles at $y=0 d_{e}$ exhibit minor differences of maximal $5 \%$ between the mesh grids. The turbulence kinetic energy profiles are estimated consistently on all grids for the shown line plots. The turbulence

kinetic energy profiles converge monotonically to the fine mesh results, where minor differences can be observed between the intermediate and fine grid at the line location at $y=0 d_{e}$. Although the line plot in the near valve region exhibits slight differences, the downstream located profiles exhibit minor differences.

The power spectra density (PSD) plots based on time-history data of the axial velocity component are shown in Fig. 5. The spectra were calculated for the signals monitored in two points, P1 and P2, sampled for all the grid resolutions ( $\mathrm{C} 1$ to $\mathrm{C} 3$ ). The locations of the probes are depicted in Fig. 1. It can be observed that using these girds the low frequency range could be consistently predicted, where the flow features are accurately captured up to about $10 \mathrm{kHz}$ on the finest grid. Thus, the numerical approach does not influence the flow scales of interest.

\section{Results}

\section{Comparison of simulations and experiments}

A direct comparison of the numerical results to experimentally measured data is required for reliable validation. The compared data are the total pressure loss calculated between the cylinder (i.e. the cutplane 0 depicted in Fig.1) and the exit of the exhaust port (i.e. the cut-plane $y=10 d_{e}$ specified in Fig. 1). The experiments have been performed for three different valve lifts, i.e. $3 \mathrm{~mm}, 4 \mathrm{~mm}$, and $5 \mathrm{~mm}$.

The comparison between the URANS, LES, and the experimentally measured total pressure losses for three different valve lifts is presented in Tab. 2. LES predicts the total pressure loss in agreement with the experimental data (differences lay within 2\%). The estimations for the total pressure loss using the URANS approach are slightly overestimated (differences roughly within 10\%) compared to the values 
obtained by LES and the experiments. Due to the complex nature of the flow in the exhaust port, which is characterized by flow separation, induced shear-layers, and stagnation regions, this is not unexpected.

\section{General flow observations}

The general characteristics of the flow field in the exhaust port are described using the data obtained from the LES calculations performed for a $4 \mathrm{~mm}$ valve lift (case C5 in Tab. 1). In Fig. 6, the time-averaged velocity magnitude contours in a cut (location is indicated in Fig. 1 by a dash-dotted line) shows the flow through one exhaust port and the exit pipe. The flow originating from the cylinder streams through the valve gap and accelerates initially due to the reduction of the cross-sectional area. The peak velocities in the valve gap are of the order of $110 \mathrm{~m} / \mathrm{s}$. However, due to the idealized, laboratory-like conditions, this velocity is rather low as compared with a real engine scenario. Downstream of the contraction, the flow expands into the exhaust port, where the cross-sectional area increases again. Due to the sudden expansion, flow separation and recirculation regions are found in the exhaust port, which can be clearly seen in Fig. 7. The presence of the valve causes an annular, high velocity, unsteady, jet-like flow structure, which is formed on the top surface of the valve (see also Fig. 7). A detailed view cutting the valve port is shown on the left top of Fig. 7, which shows that the flow exhibits a jagged annular shape. Due to Görtler-like vortical structures forming at the valve wall, which are caused by the curvature of the valve, the flow does not completely attach to the valve surface. The high-velocity flow follows the valve stem into the port and then reaches the top of the valve stem,

impinging on the larger curvature radius port channel wall. After the impingement onto the top wall of the first port channel, the flow streams along the outer bend of the port into the exit pipe, directed by the curvature of the exhaust port.

Due to the curvature of the geometry in the exhaust port, the high velocity fluid impinges onto the outer wall. Further, two Dean-like counter-rotating vortices are captured right downstream of the valve in the LES calculations, as shown in Fig. 8. Due to the effect of the irregular port shape and the valve stems, the two counter rotating vortices are not symmetric.

Generally, the flow exhibits similar flow field characteristics in both exhaust ports. However, Fig. 1 shows that the curvature and the length, from the cylinder to the conjunction, for the two exhaust ports are different. Due to the higher curvature radius in valve $4 \mathrm{~b}$ the flow follows smoothly the exhaust port walls, whereas the flow streaming through valve 4a hits the top wall at high velocities and therefore, secondary flow structures are induced before the stream flows into the exit pipe. This can be seen in Fig. 9 illustrating the streamlines, a high velocity magnitude peak close to the top wall of valve port $4 \mathrm{a}$ 
is observable, which does not occur at this magnitude in valve port $4 \mathrm{~b}$. Moreover, the observed flow velocities are higher in the valve port $4 \mathrm{a}$ than in the valve port $4 \mathrm{~b}$. At the entrance of both valve ports acts the same pressure from the cylinder and at the location where the valve ports merge the static pressure is also equal. Since, the same pressure difference acts over each valve port, whereas the streaming length is shorter in valve port $4 \mathrm{a}$ than in valve port $4 \mathrm{~b}$, the pressure gradient is higher in valve port $4 \mathrm{a}$ than in valve port $4 \mathrm{~b}$. Due to the higher pressure gradient, the flow in valve port $4 \mathrm{a}$ is accelerated to higher velocities than the flow in valve port $4 \mathrm{~b}$.

The two streams originating from the two channels join in the exit pipe and interact with each other. A secondary helical flow structure is generated in the exit pipe, which can be clearly seen by the streamline visualization shown in Fig. 9. The detail furthest to the right in Fig. 9 shows that the flow streaming through valve port $4 \mathrm{~b}$ do not exhibit the strong helical motion before the conjunction as the helical flow in the exit pipe. However, the flow through valve port 4a streams towards the top wall and deflects there into the exit pipe. In the valve port, the annular jet stream is surrounded by low velocity fluid, which is indicated by the coloring of the streamlines by the velocity magnitude in Fig. 9. From this low velocity region, the fluid is drawn into the exit pipe due to fluid inertia and a low pressure region establishes (see Fig. 11). The stream coming from the valve port $4 \mathrm{~b}$ has the lower momentum than the stream originating from valve port $4 \mathrm{a}$ and therefore, the stream from valve port $4 \mathrm{~b}$ is deflected around the stream originating from valve port 4a. The redirected stream flows towards the low pressure region generated at the junction between the exit pipe and valve port $4 \mathrm{a}$, which initiates the helical motion. This is clearly visible in the mid detail shown in Fig. 9.

Figure 1 illustrates that the valves are located closely to the outer periphery of the cylinder. Moreover, a part of the exhaust port geometry is cut to not jut over the cylinder periphery. The consequences to the flow field can be seen in Fig. 6 in the cross-section 1. A small low velocity region at the back side of the valve towards the cylinder wall can be noted (coordinate axis $x$ is indicated to the left of the cross-section). Thus, the close positioning acts as an area restriction for the flow.

The flow field develops similar for the simulated valve lifts. Figure 10 shows a streamline visualization for all the three valve lifts. The potential core length of a jet is proportional to the extensions of the orifice. Thus, the core region of the annular jet increases for larger valve lifts and the flow momentum reaches further into the exhaust port. The wider annular jet fills more volume of the valve port, and therefore the separation zones decrease in size. 


\section{Total pressure losses}

In order to illustrate where the main total pressure losses occur, the area-weighted total pressure values have been calculated along several cross-sectional surfaces. The locations of each of the crosssections involved ( 0 to 6$)$ are the same as shown in Fig. 11. The total pressure differences between two consequent cross-sectional planes are listed in Tab. 3 for a valve lift of $5 \mathrm{~mm}$. As expected, the major total pressure loss takes place in the port and not in the exit pipe. The highest total pressure loss of $47.9 \%$ for the LES simulations manifests between the cross-planes 1 and 2 . The total pressure loss between the cylinder, cross-plane 0 , and cross-plane 1 is $27.1 \%$, which is significantly lower. Contrary, the URANS approach predicts a higher total pressure loss between the cross-planes 0 and 1 and a lower pressure loss between the cross-planes 1 and 2. This observed trend is similar for all other simulated valve lifts.

The total pressure contours obtained by the two simulation approaches is shown in Fig. 11 and the illustration clarifies the origin of the differences. Already in Tab. 3 it is observable that the total pressure in the cylinder is slightly higher predicted with the URANS approach, although the same boundary conditions have been applied for both simulations. Thus, a flow restriction has developed in the computational domain with the URANS approach, which provokes that a higher in-cylinder total pressure is accumulated. Nevertheless, the turbulence closure used for the URANS simulation clearly overestimates the boundary layer separation in the gap formed between the valve head and the valve seat. There, a low static pressure region develops due to the Venturi effect of a consequent contraction and expansion. The low pressure zone is compensated with an overestimation of turbulent viscosity in the URANS simulation, since the simple turbulence closure model cannot handle the compressible flow effect. However, the severe separation at the valve seat leaves a much more narrow channel for the flow. Thus, the flow is hindered to stream into the valve port, which generates high total pressure losses before the cross-plane 1. As a consequence, the flow velocities develop much lower downstream of the narrow gap. Only minor flow separation takes place in between the cross-planes 1 and 2, which explains the low estimated total pressure loss in this segment compared to the total pressure loss predicted by the LES simulations. Further downstream, the total pressure contours develop rather smooth compared to the LES computed total pressure contours. Thus, the overestimation of the separation has remarkable consequences on the entire flow field downstream.

For the LES data, the flow remains initially attached on the valve seat and separates form the valve and the valve port due to the geometrical sharp edges formed by the valve seat. The valve seat is located upstream of cross-plane 1. Due to the longer attached flow, much higher flow velocities are observed in 
the LES data than for the URANS data. This induces larger gradients of the total pressure throughout the entire domain downstream. Nevertheless, the more severe separation occurs downstream of cross-plane 1.

The low total pressure region in the valve port $4 \mathrm{a}$ is predicted differently by URANS and LES. The LES predicts a significant lower total pressure region in the valve port $4 \mathrm{a}$ than in the valve port $4 \mathrm{~b}$, which implies that the total pressure losses are much higher in valve port $4 \mathrm{a}$ than in valve port $4 \mathrm{~b}$. Further, the low total pressure region predicted by the LES simulations stretches a significant amount into the exit pipe, where the flow field in the downstream connected manifold could be influenced. Therefore, the helical motion in the exit pipe is predicted differently with the two approaches.

\section{Flow structures and losses in the exhaust port}

There are several mechanisms causing a total pressure losses in a pipe system, as e.g. expansion, friction, or centrifugal mechanisms. In applied fluid mechanics, empirical formulas are often used for the estimations of the total pressure losses due to different mechanisms. The empirical formulas are based on certain assumptions for a specific type of flow. By applying the empirical formulas to a complex flow, the assumptions might be violated. Therefore, it is only reasonable to use these empirical formulas to calculate an estimation of the pressure loss values. By comparing the estimation with the simulation data in a certain section, the major source of total pressure loss in the particular section can be identified.

The geometry of the cylinder together with the port seat and the exhaust valve generate an annular nozzle, in which the flow is accelerated. The flow separates from the walls in the divergent section, which leads to a total pressure loss larger than the equivalent value for a straight pipe. Lighthill [14] has pointed out that for a generalized contraction, the entire pressure drop is not converted to kinetic energy (and hence, recoverable at a subsequent enlargement). Thus, the irreversible components of the pressure drop must be considered, which stem from dissipation of energy and from the induced secondary flow motion, since these are sources of total pressure loss.

A contraction model with $90^{\circ}$ cone angle is used to estimate the total pressure loss resulting in the near region of the contraction. Since the variation of the density is rather small (maximum Mach number is about 0.3 ) for the cold simulated case, the flow is idealized as incompressible for this estimation. With these simplifications, Bernoulli's equation can be used here and the total pressure loss for a contraction 
can be estimated by [15],

$$
\Delta p_{C_{t o t}}=K_{C} \cdot \rho \frac{V_{g a p}^{2}}{2}
$$

where $\rho$ is the density of the streaming media, and $K_{C}$ is the resistance coefficient and depends on the area ratio. The area ratio is considered to be 15 . Thus, $K_{C}$ can be estimated as 0.19 according to the charts available in handbooks [15]. The velocity in the gap $V_{\text {gap }}$ varies between the values 105 and 110 $\mathrm{m} / \mathrm{s}$. The empirical estimation of the total pressure loss is between $1.26 \mathrm{kPa}$ to $1.38 \mathrm{kPa}$, which is of the same order of magnitude as the total pressure loss listed in Tab. 3 for the LES data, between the cross-planes 0 and 1.

The flow experiences the Venturi effect when passing through the narrow gap, which is formed by the exhaust port and valve. Hence, the flow is initially accelerated (a locally low pressure region develops in the narrowest cross-section) within the valve gap. Further downstream within the exhaust port, the flow is decelerated due to the increase in the cross-sectional area, which is accompanied by a rise of the static pressure. The dissipation of energy caused by the developed secondary flow structures leads to the fact that the total kinetic energy is not entirely converted into pressure energy. The cone angle for the enlargement here is obviously larger than $45^{\circ}$. Hence, a sudden enlargement model can be employed to estimate the total pressure drop caused by this enlargement [16]. The total pressure loss caused by the expansion mechanism is accordingly to Robert [15],

$$
\Delta p_{E_{t o t}}=\rho \frac{V_{g a p}^{2}}{2}\left(1-\frac{A_{1}}{A_{2}}\right)^{2}
$$

where the area ratio $A_{1} / A_{2}$ can be considered to be 0.5 between the cross-planes 1 and 2 (the locations are indicated in Fig. 6). The total pressure loss can be approximated between 1.65 to $1.82 \mathrm{kPa}$. This result and the total pressure loss tabulated in Tab. 3 have the same order of thousands Pascal. Hence, the total pressure drop generated between the cross-planes 1 and 2 mainly results due to the expansion of the geometry.

Figure 10 shows the streamlines visualizations at different valve lifts. On can separate the flow field in two zones: a high velocity region and another low velocity region. As the valve lift increases, the high velocity region, representing the annular jet, becomes larger and the flow recirculation zones become smaller. Thus, the total pressure loss caused by expansion mechanism depends on the valve lift.

The distribution of the Wall Shear Stress (WSS) magnitude has been calculated for a valve lift of 4 $m m$ (C5) and is depicted in Fig. 12. The flow interactions with the solid walls of the exhaust port are 
indicated by this quantity. Thus, wall friction losses are generated, which represent another source of the total pressure loss. Figure 12 shows three locations on the exhaust port geometry, where peak values for the WSS occur, indicated by 1,2, and 3. Downstream of the valve gap, the annular jet-like flow contracts towards the valve stem and impinges with high velocity onto the valve stem. The interaction between the high velocity flow and the valve stem causes friction forces and a momentum loss (outlined as 1 in Fig. 12). However, the high speed jet causes primary mechanical loads on the valve stem. In the near region of the valve gap, large WSS magnitudes on both the seat and the edge of the valve manifest due to the high velocities through the valve gap (named 2 in Fig. 12). The highest WSS values can be observed in the region, where the valve ports are closely positioned to the outer periphery of the cylinder. The locally high velocities are caused by the area restriction due to the cut of the initial valve port. The consequences onto the flow field in the valve port have been described in the previous section. In the front detailed view of the valves shown on the top left of Fig. 12, vertical streaks can be observed, which stem from the Görtler-like vortical structures as described in the previous section.

The total pressure loss caused by friction forces can be then calculated using the equation [17]:

$$
\Delta p_{F_{\text {tot }}}=\frac{\iint_{A_{\text {wall }}} \tau_{w} d A_{\text {wall }}}{A_{\text {eff }}}
$$

where $\tau_{w}$ is the WSS acting on the wall area segment $A_{e f f}$, which is the effective (projected) area normal to the streamwise direction. Due to the irregular shape of the exhaust port geometry and the existence of the valve, the accurate calculation of the effective area is challenging. The estimation used is an average of several cut planes through the exhaust port geometry. The resulted total pressure loss due to wall friction is $268 P a$ in the whole exhaust port (denoted by 4 in Fig. 1). Thus, the total pressure loss caused by friction is on the order of hundreds of Pascals. Thus, the wall friction is not the main source of the total pressure loss in an exhaust port.

The total pressure loss for bended pipes due to the bending can be estimated by the equation [15]:

$$
\Delta p_{B_{t o t}}=K_{b} \cdot \rho \frac{V^{2}}{2}
$$

where $V$ is the mean streamwise velocity of $40 \mathrm{~m} / \mathrm{s}$, calculated as area-weighted average velocity at the cross-plane number 3 in Fig. $6 . K_{b}$ is the loss coefficient in the bend, which depends on the bend angle and the ratio between centerline radius of the bend and the internal diameter of the pipe [15]. The bend angle is assumed to be $90^{\circ}$. The centerline radius of the bend, following the entire geometry from the head of the cylinder to the centerline of the exit pipe, was estimated as $75 \mathrm{~mm}$. The pipe shape is rather 
complex in the bend and the area changes over the bend pipe length. However, the internal pipe diameter is assumed to be the diameter of the exit pipe, $d_{e}=42 \mathrm{~mm}$. Thus, the ratio between the centerline radius of the bend and the internal diameter of the pipe is approximately 1.78 . The loss coefficient $K_{b}$ can be then identified to be 0.3 from the bend loss coefficient chart for a pipe [15]. The total pressure loss is 288 $P a$. The total pressure loss caused by the end pipe is on the order of hundred Pascals. This contribution is an important part of the total pressure loss in the exhaust port, but not a major component.

\section{Discussion and conclusions}

LES and URANS (using the standard $k-\varepsilon$ closure model) flow calculations were performed in order to quantify the flow field and the kinetic energy losses associated with the exhaust port geometry of a heavy duty Diesel engine. The calculations were carried out for three different exhaust valve lifts. However, the differences for the small variations of the valve lift simulated did not significantly change the flow field. It was found that unsteady, relatively large vortical scales dominate the flow in the exhaust port. The comparisons between the LES, URANS, and provided experimental data in terms of the total pressure loss suggested that the LES predicts the behavior of the flow in such a complex $3 \mathrm{D}$ geometry more accurately.

Evaluating the total pressure loss in between segments of the exhaust port showed that about $70-$ $80 \%$ of the total pressure loss occurs in the near gap region of the exhaust valve and originates from the contraction and the enlargement process of the flow for small valve lifts. The energy dissipated through flow separation and induced secondary flow motion is the main source for the total pressure losses. In the first two segments, between cylinder to maximum contraction and maximum contraction to expansion, the two simulations approaches, i.e. LES and URANS, disagreed significantly in the prediction of the total pressure loss. The LES data exposed the major losses for the expansion process, while URANS data showed the most losses in the contraction segment. The main difference in the flow field stems from the intensity of the flow separation predicted by the approaches. Where the URANS predicted drastic separation at the entrance of the valve seat channel between the valve and the valve port.

The $k-\varepsilon$ turbulence closure model relays on a wall damping function, which seams to allow too much dissipation at the gap walls, since the stratification leads to high anisotropy of the shear stress, due to the relatively high pressure gradient, which is not taken into account. Therefore, the flow separation is predicted too severe. This issue is specific to the $k-\varepsilon$ closure model used and is not globally valid for all URANS approaches. However, this draw-back of the $k-\varepsilon$ closure model results in errors for this specific problem, which might be no issue for other areas of application, e.g. at larger valve lifts, the 
turbulence closure model might deliver reasonable results. However, a more sophisticated modeling of the RANS closure, as e.g. including inhomogeneous or non-isotropic turbulence, might lead to a more accurate results. Such models accounting for the anisotropy are the nonlinear Eddy-Viscosity Models (EVM) or the Reynolds stress models (RSM), where the eddy viscosity is modeled in a more advanced manner or the Reynolds stresses are directly computed. Nonetheless, these modeling is computationally more expansive. This illustrates that the turbulence closer model needs be chosen appropriately to the problem.

The simulations were performed on a cray XE6 system Lindgren at PDC. The URANS approach used requires two additional equations for turbulence closure compared to the set of equations used within LES. Therefore, the numerical effort per time-step increases form approximately $55 s$ (LES) to approximately $70 s$ with URANS on 32 cores. However, the statistical converged data could be achieved after about $50 k$ iterations using URANS, while about $400 k$ iterations have been required using LES. Hence, the total computational effort is higher with LES than URANS. Moreover, the same mesh grid has been used for both, LES and URANS. However, the turbulence modeling used by URANS permits to use less fine grids than required for LES. Hence, a grid optimized for URANS (with reduced cell count) would lead to an additional speed up for these simulations.

It was shown that the flow separation induces the largest proportion of the total pressure losses, which occurs due to the relatively sharp opening angle of the port geometry. To decrease the energy loss or total pressure loss within this process, it would be relevant to design a more gradual, smooth change of the distance of the gap to decrease the effect of initial separation.

The flow velocity in the gap between valve head and valve seat enters into the total pressure loss estimations of contraction and enlargement quadratically. Therefore, lowering the velocity in the gap is an effective method to decrease the total pressure loss. Due to the continuity equation, the velocity in the gap is decreasing when the flow area increases. Hence, the possible way to decrease the total pressure loss is to adjust the valve lift timing. Increasing the flow area in a short time, would lead to a shorter time period where flow reaches high velocities in the valve gap. However, changing the valve timing might lead to disadvantages regarding the emission production in the cylinder.

Due to the different streaming length through the ports and the same driving pressure difference acting on the flow, the pressure gradient in the shorter valve port is larger than in the longer valve port. Hence, the flow velocities in the shorter valve port were observed to be higher. As mentioned in the previous paragraph, many sources of the total pressure losses scale quadratically with the occurring flow velocities. Thus, equal length of the valve ports should be considered to equilibrate the manifesting 
velocities between the ports, in order to minimize the losses.

The valve ports are located as close to the periphery of the cylinder as possible due to geometrical constrains. However, this results in a flow interaction where the flow is accelerated close to the cylinder walls. With the high velocities close to the cylinder walls, high WSS are induced, which generate a flow resistance. This leads to a reduced streaming area through the valve port.

The flow in the valve port designed with a higher curvature radius towards exit pipe exhibited significantly lower total losses, than the flow streaming through the valve port with lower curvature radius. The strong curvature leads to a rough impingement of the annular jet-like structure on the top wall of the valve port. Hence, the induced secondary flow motion dissipates the otherwise reusable amount of the flow kinetic energy. Therefore, the valve ports should be designed with the largest curvature radius as possible to avoid losses.

\section{Acknowledgements}

This work was supported by the Competence Center for Gas Exchange (CCGEx) at KTH and the China Scholarship Council (CSC). The computational resources provided through the Swedish National Infrastructure for Computing (SNIC 2013/1-72) are greatly acknowledged.

\section{References}

[1] Baines, N. C., 2005. Fundamentals of Turbocharging. Concepts NREC, Vermont USA.

[2] Galindo, J., Serrano, J., Climent, H., and Tiseira, A., 2008. "Experiments and modelling of surge in small centrifugal compressor for automotive engines". Experimental Thermal and Fluid Science, 32(3), January, pp. 818-826.

[3] Caton, J., and Heywood, J., 1981. "An experimental and analytical study of heat transfer in an engine exhaust port”. International Journal of Heat and Mass Transfer, 24(4), pp. 581-595.

[4] Harrington, J. W., 1979. "Visualization of the flow of exhaust through an internal combustion engine exhaust port”. PhD thesis, Massachusetts Institute of Technology, Department of Mechanical Engineering.

[5] GammaTechnologiesInc., 2012. “Flow Theory Manual”. In Tech. Report.

[6] Danov, S., 1997. "Identification of discharge coefficients for flow through valves and ports of internal combustion engines”. In SAE Technical Paper 970642.

[7] Onorati, A., Cerri, T., Ceccarani, M., and Cacciatore, D., 2005. "Experimental Analysis and 1D 
Thermo-Fluid Dynamic Simulation of a High Performance Lamborghini V10 S.I. Engine”. In SAE Technical Paper 2005-24-081.

[8] Han, Z., and Reitz, R., 1995. "Turbulence modeling of intrnal combustion engines using rng $k-\varepsilon$ models". Combustion science and technology, 106, pp. 267-295.

[9] CD-Adapco, 2011. STAR-CCM+ USER GUIDE, version 6.06.017 ed. The American Society of Mechanical Engineers.

[10] G.Margolin, L., Rider, W. J., and Grinstein, F. F., 2006. "Modeling turbulent flow with implicit les". Journal of Turbulence, 7, pp. 1-27.

[11] Garmann, D. J., Visbal, M. R., and Orkwis, P. D., 2013. "Comparative study of implicit and subgrid-scale model large-eddy simulation techniques for low-reynolds number airfoil applications". International Journal for Numerical Methods in Fluids, 71(12), pp. 1546-1565.

[12] Semlitsch, B., JyothishKumar, V., Mihaescu, M., Fuchs, L., Gutmark, E., and Gancedo, M., 2014. "Numerical flow analysis of a centrifugal compressor with ported and without ported shroud". In SAE Technical Paper 2014-01-1655.

[13] Wilcox, D. C., 2006. Turbulence Modeling for CFD. DCW Industries, California, USA.

[14] Lighthill, J., 1986. An Informal Introduction to Theoretical Fluid Mechanics. Oxford University Press., Oxford, UK.

[15] Robert, L. M., 2005. Applied Fluid Mechanics. Pearson Prentice Hall, Oxford, UK.

[16] Ghose, S., and Kline, S. J., 1978. "The computation of optimum pressure recovery in twodimensional diffusers". Trans A.S.M.E., Journal of Fluid Engineering, 100, pp. 419-426.

[17] Munson, B. R., 2012. Fundamentals of Fluid Mechanics. Hoboken, NJ: Wiley and Sons. 


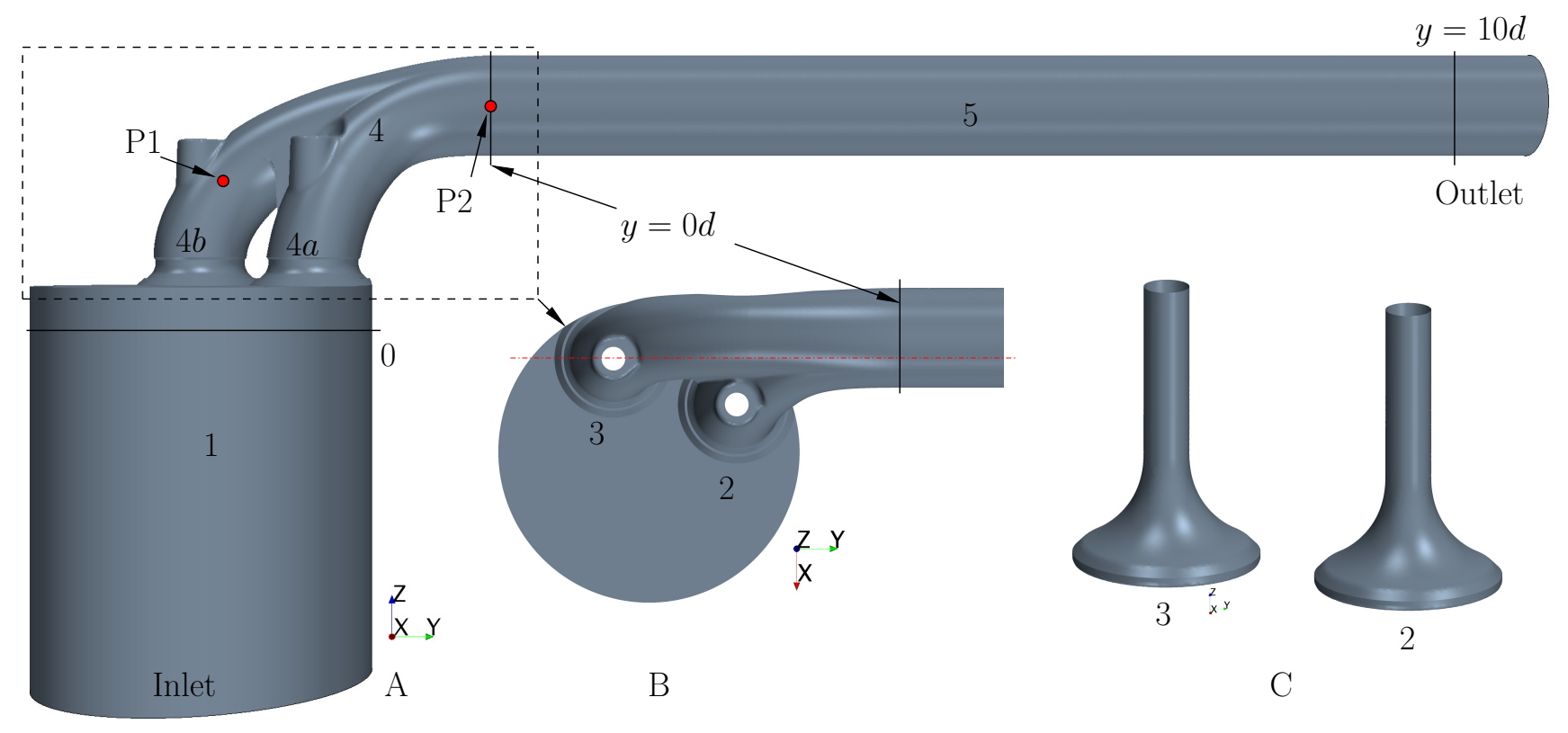

Fig. 1. Details of the considered ICE cylinder and exhaust ports geometry. A: the front view; B: the top view; C: isometric view of the two valves 3. 1: cylinder; 2 and 3: valves; 4: exhaust port; 5: exit pipe.

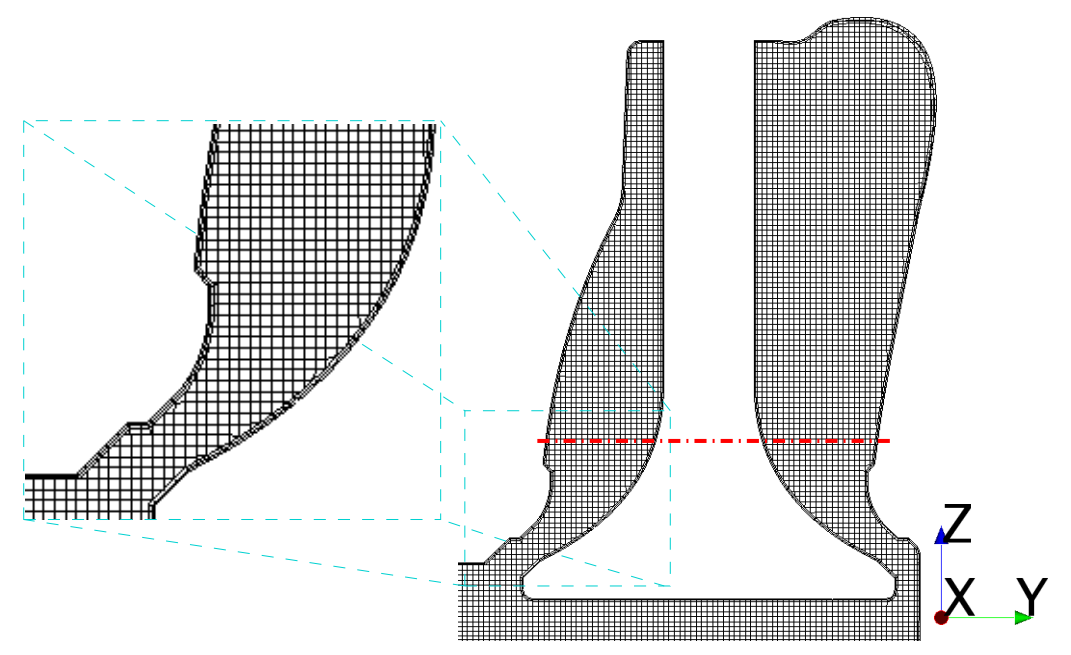

Fig. 2. An example of the computational grid is shown in the close up section around the valve 2 . 


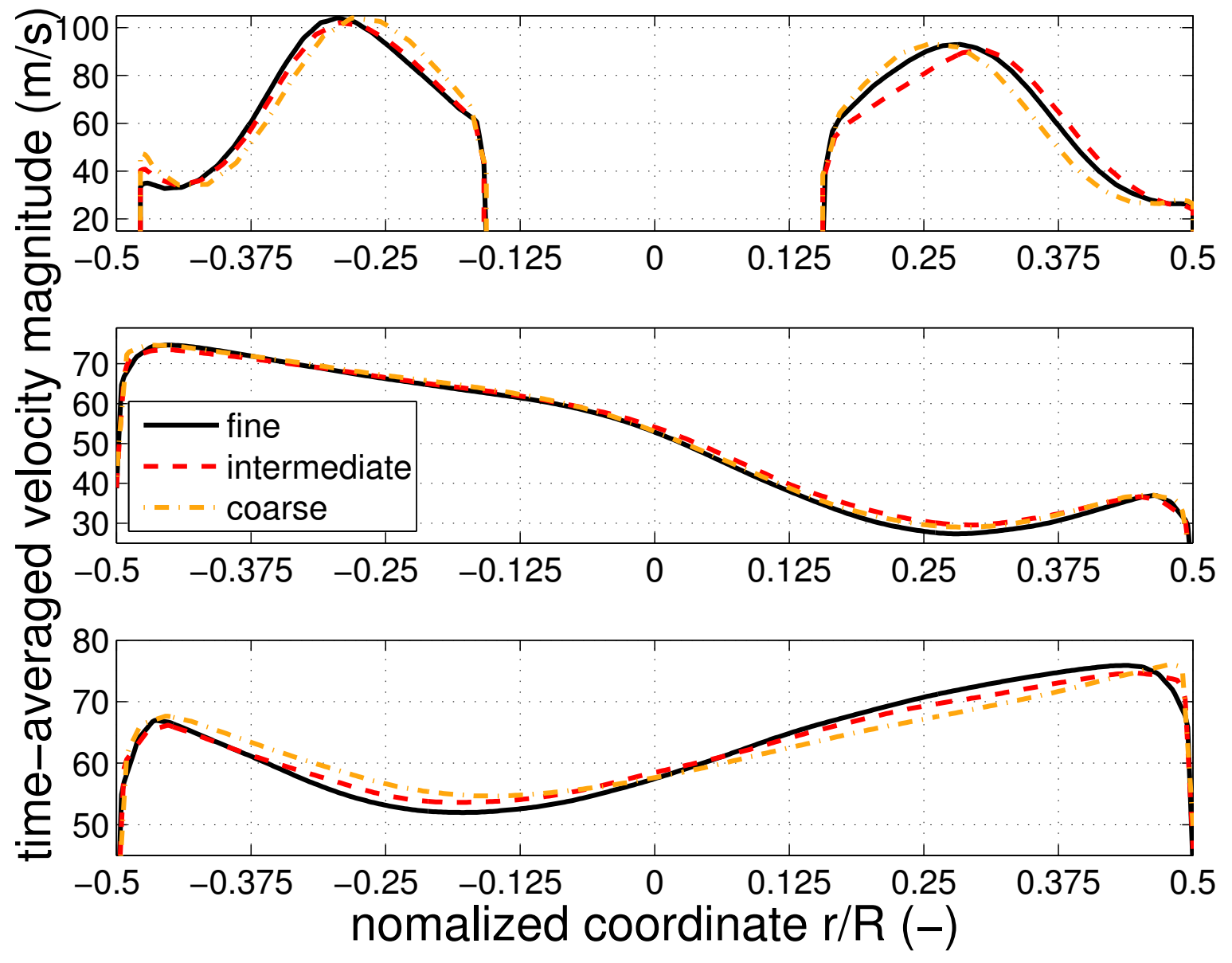

Fig. 3. Grid sensitivity study: the time-averaged velocity magnitude profiles along a horizontal line in the near valve gap region (top), on a vertical line (bottom), and a horizontal line (mid) at $y=0 d_{e}$. See Fig. 2 and Fig. 1 for indication of the locations. 


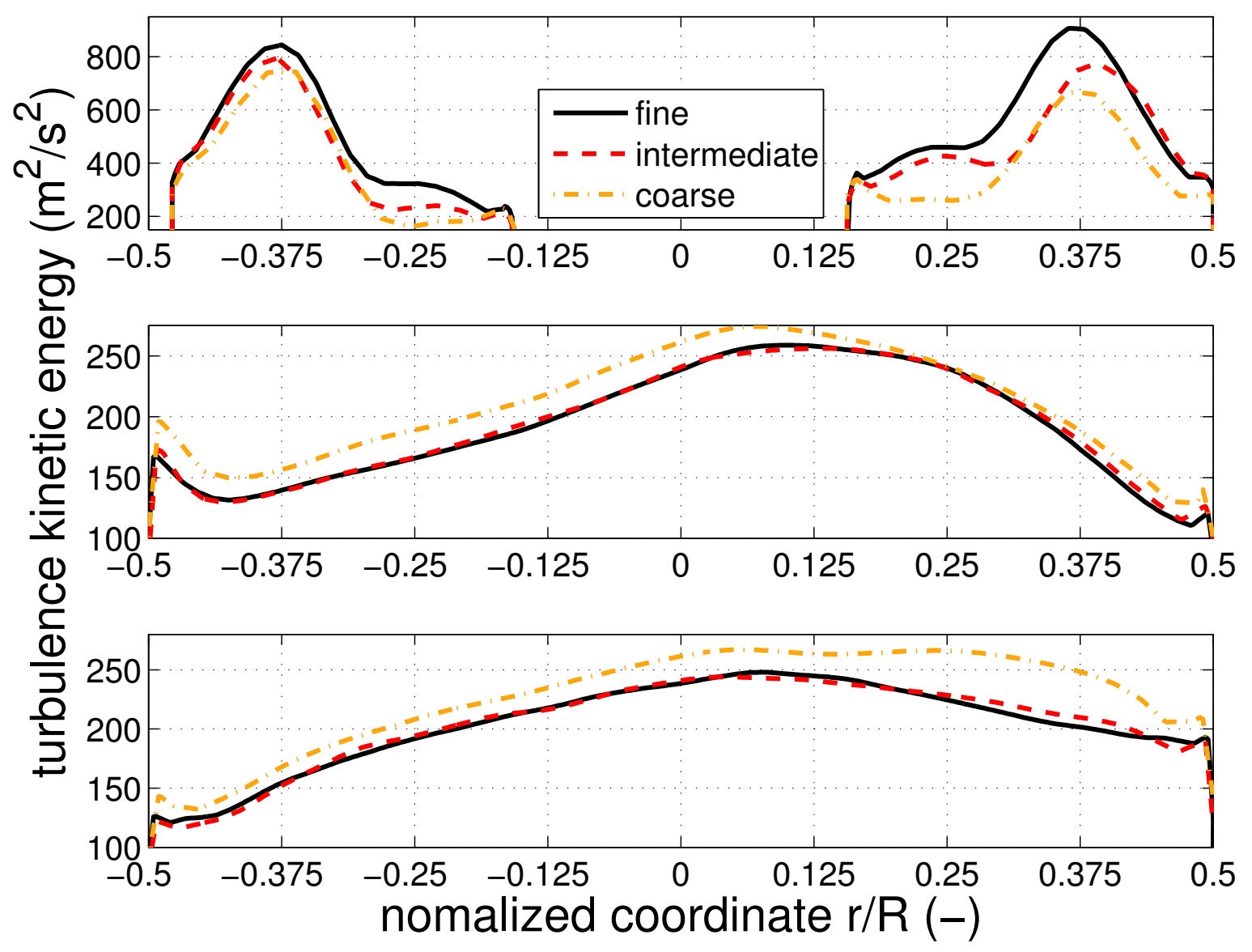

Fig. 4. Grid sensitivity study: the turbulence kinetic energy profiles along a horizontal line close to the valve gap (top), and a horizontal (mid) and a vertical (bottom) line at $y=0 d_{e}$ (see Fig. 2 and Fig. 1 for the locations) are plotted. 

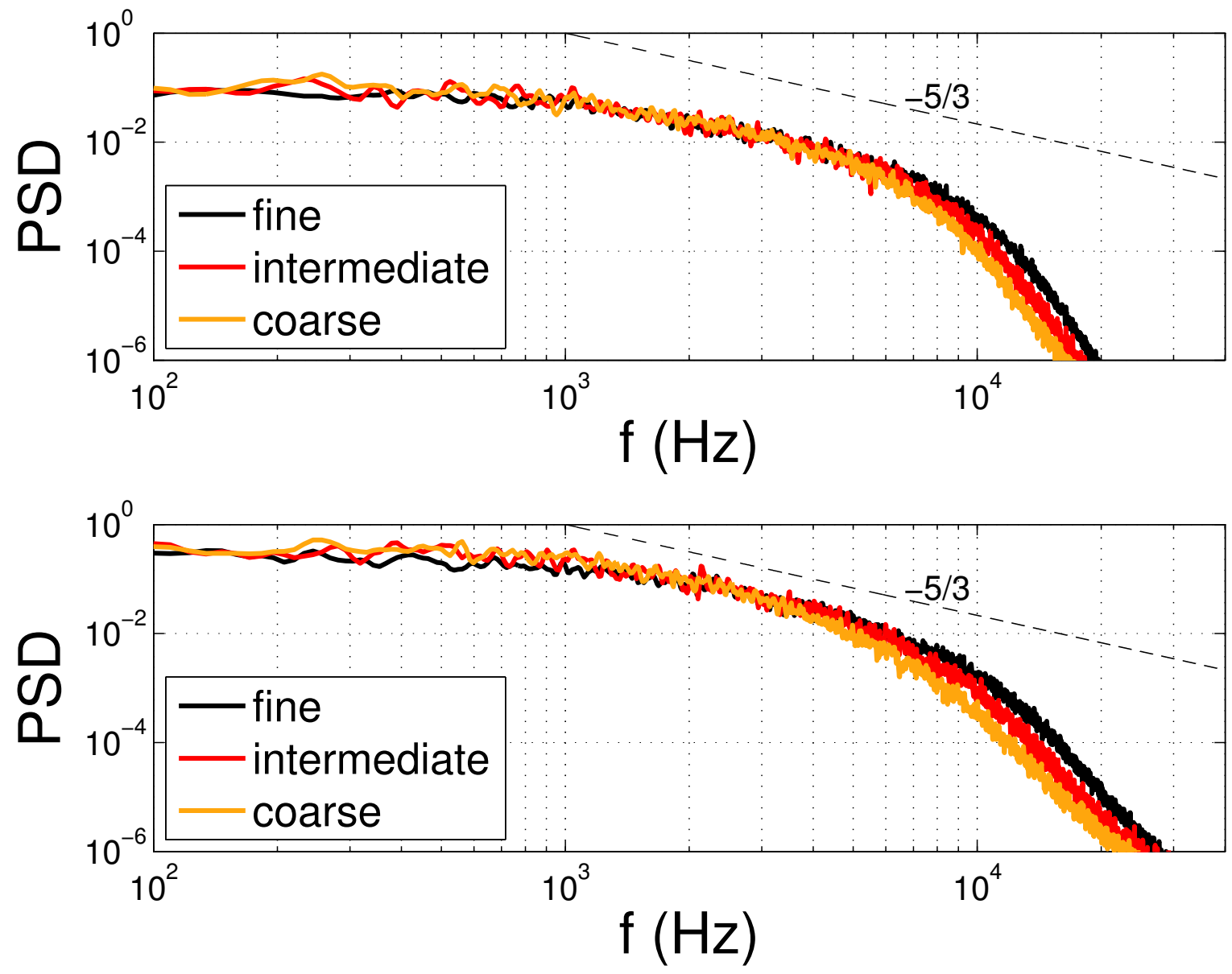

Fig. 5. The power spectra density of the axial velocity component signal monitored in probe P2 (top) and probe P1 (bottom) are show). The location of the probes P1 and P2 are indicated in Fig. 1.

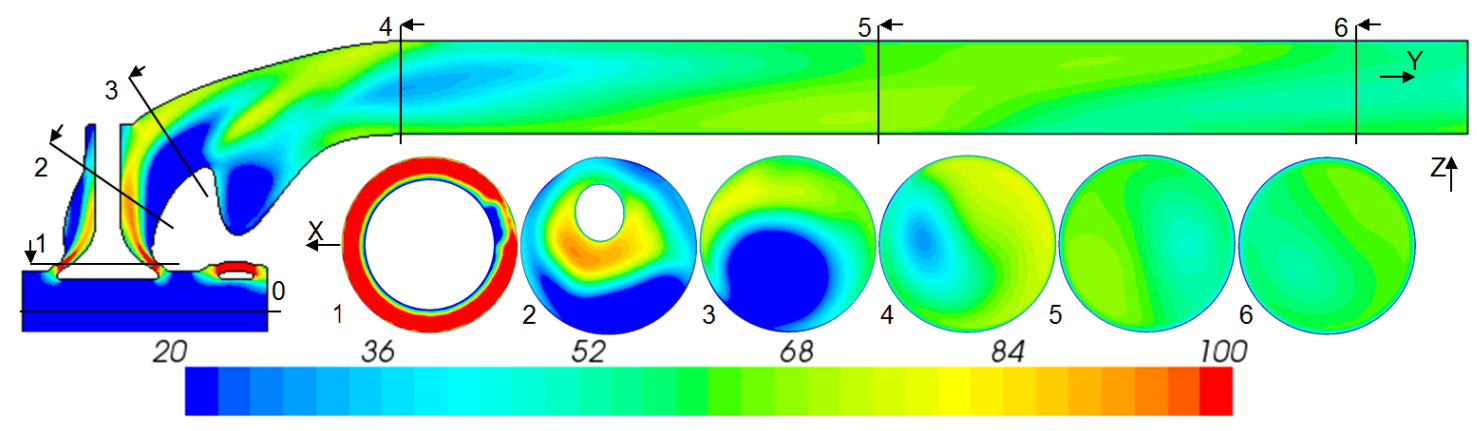

Fig. 6. The time-averaged velocity magnitude $(\mathrm{m} / \mathrm{s})$ contours obtained by the LES are shown for case C5. 


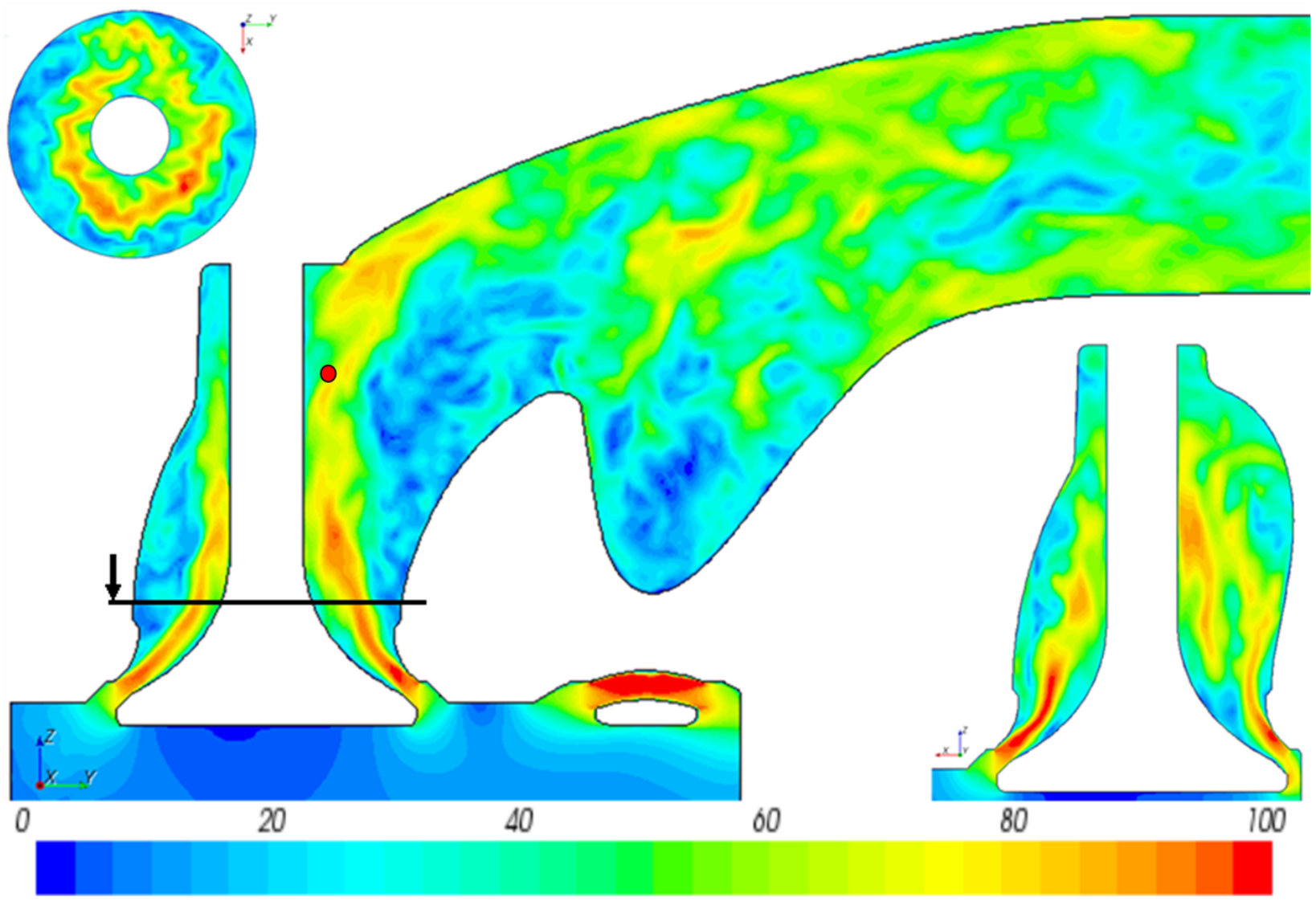

Fig. 7. An instantaneous snapshot of the LES simulation illustrates the flow field in terms of the velocity magnitude $(m / s)$ for case C5. 


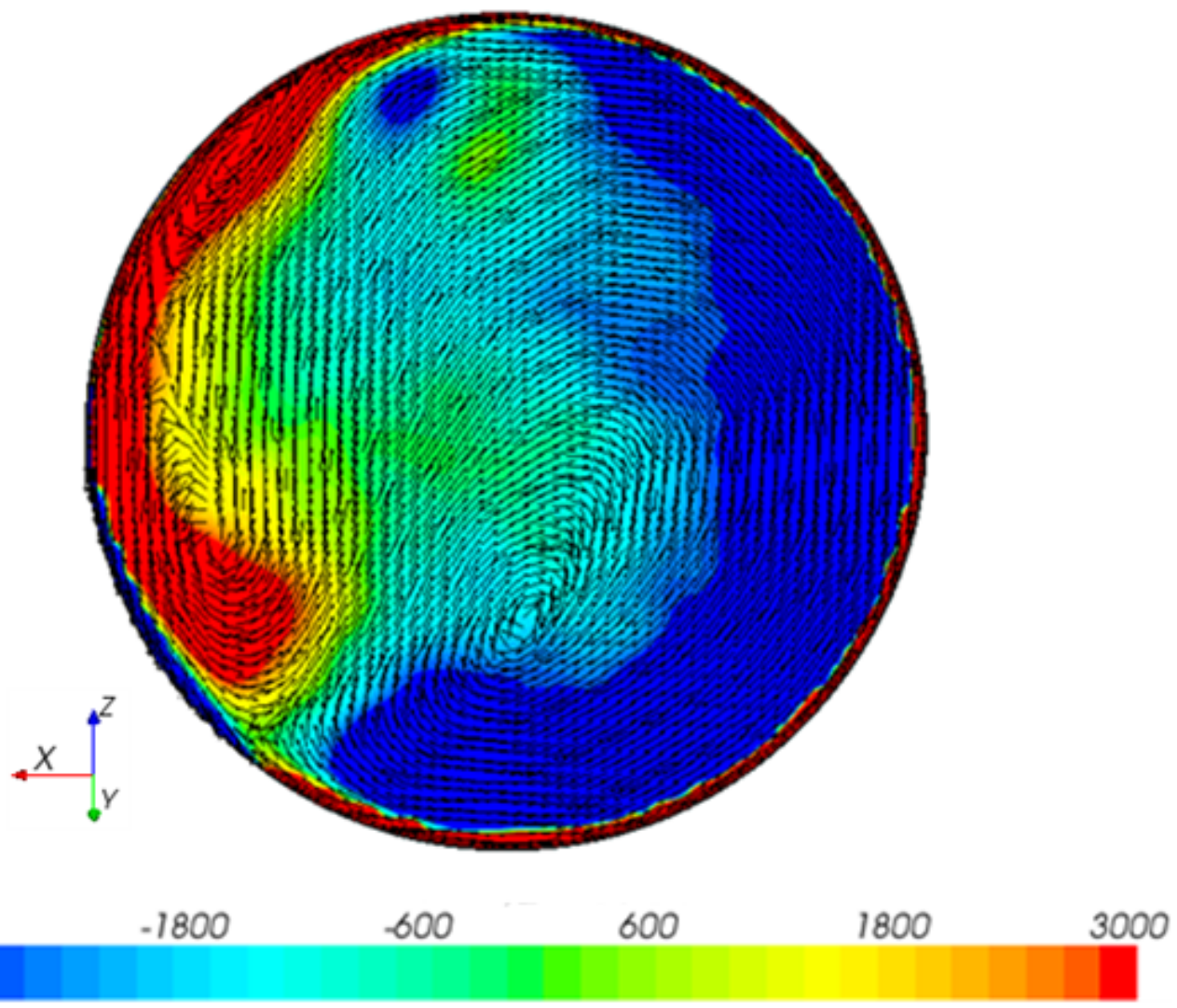

Fig. 8. The time-averaged streamwise vorticity distribution in the cross-section 3 is shown, which is indicated in Fig.6. On top, the in-plane time-averaged vorticity vectors are overlaid.

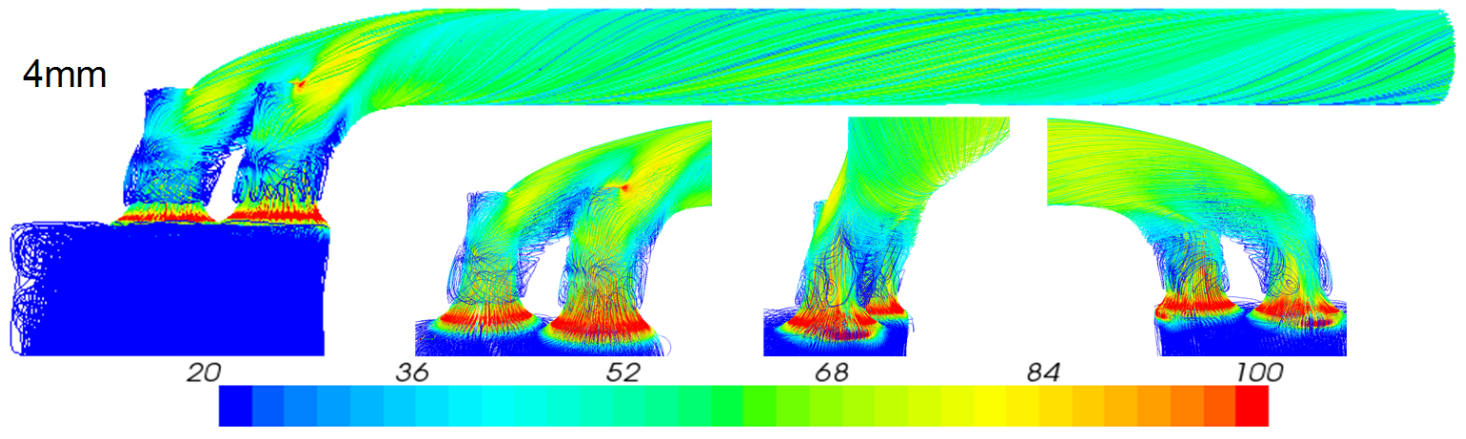

Fig. 9. Streamlines for the case with a valve lift of $4 \mathrm{~mm}$ (C5). The streamlines are colored by the time-averaged velocity magnitude $(m / s)$. Note the recirculation region surrounding the valve region, downstream of the gap between the valve and the valve seat. 


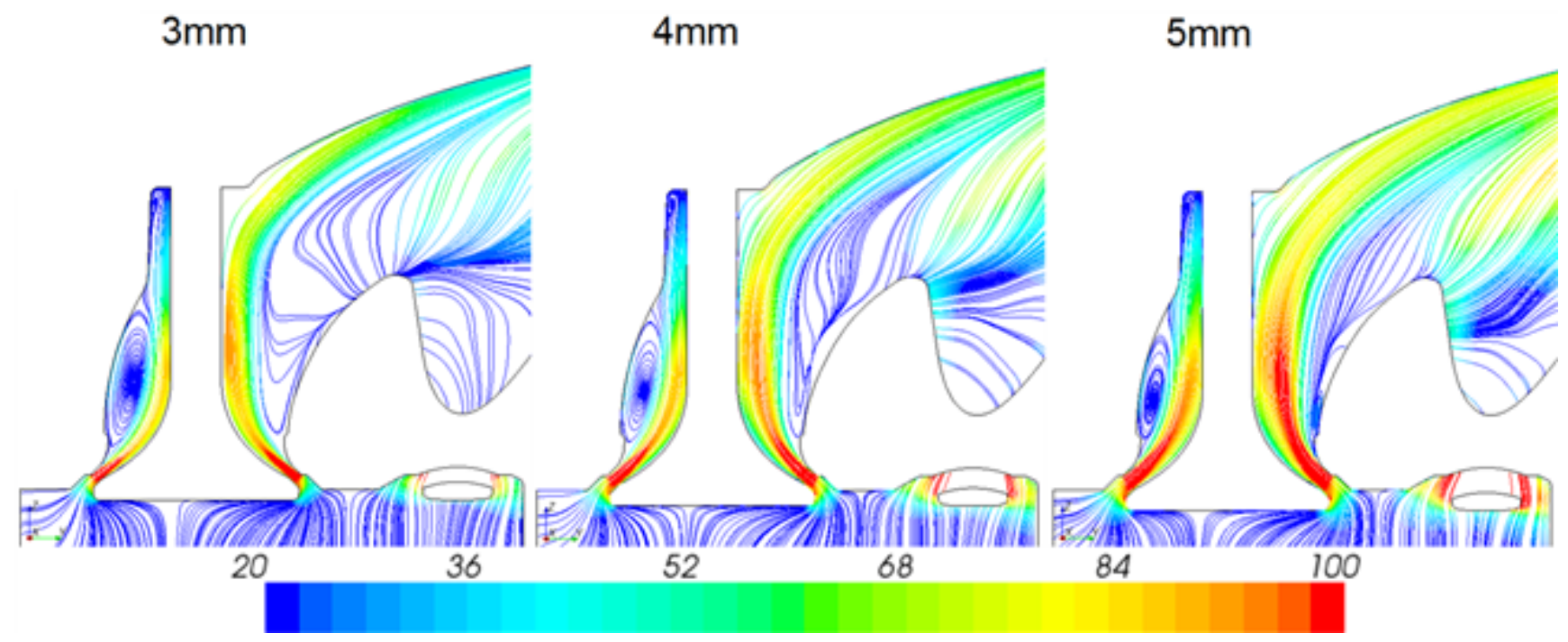

Fig. 10. The streamlines of the time-averaged LES simulation data in the exhaust port are shown for different valve lifts (i.e. $3 \mathrm{~mm}, 4 \mathrm{~mm}$, and $5 \mathrm{~mm})$. The streamlines are colored by the time-averaged velocity magnitude $(\mathrm{m} / \mathrm{s})$.
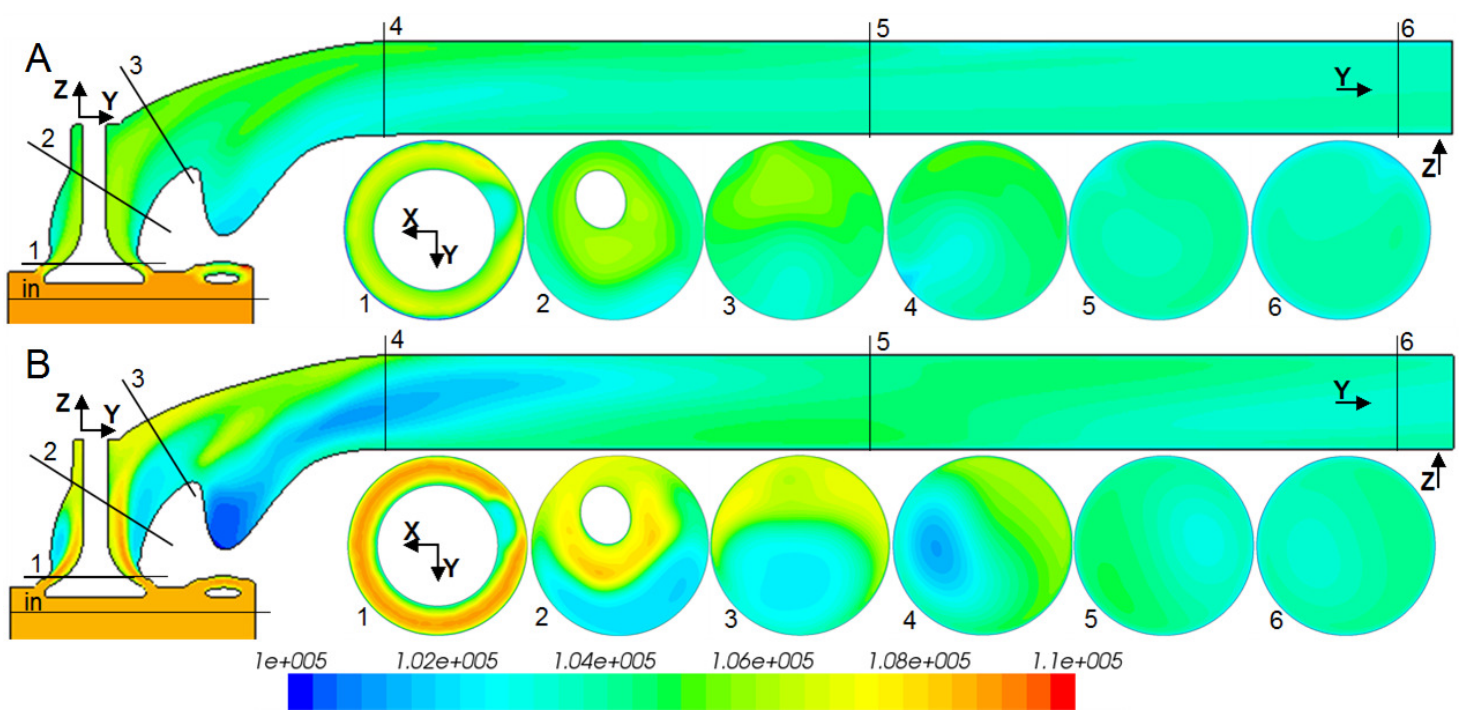

Fig. 11. The total pressure $(\mathrm{Pa})$ contours obtained by the URANS $(\mathrm{A})$ and the LES $(\mathrm{B})$ simulation approaches are shown for the case of 5 $m m$ valve lift. 


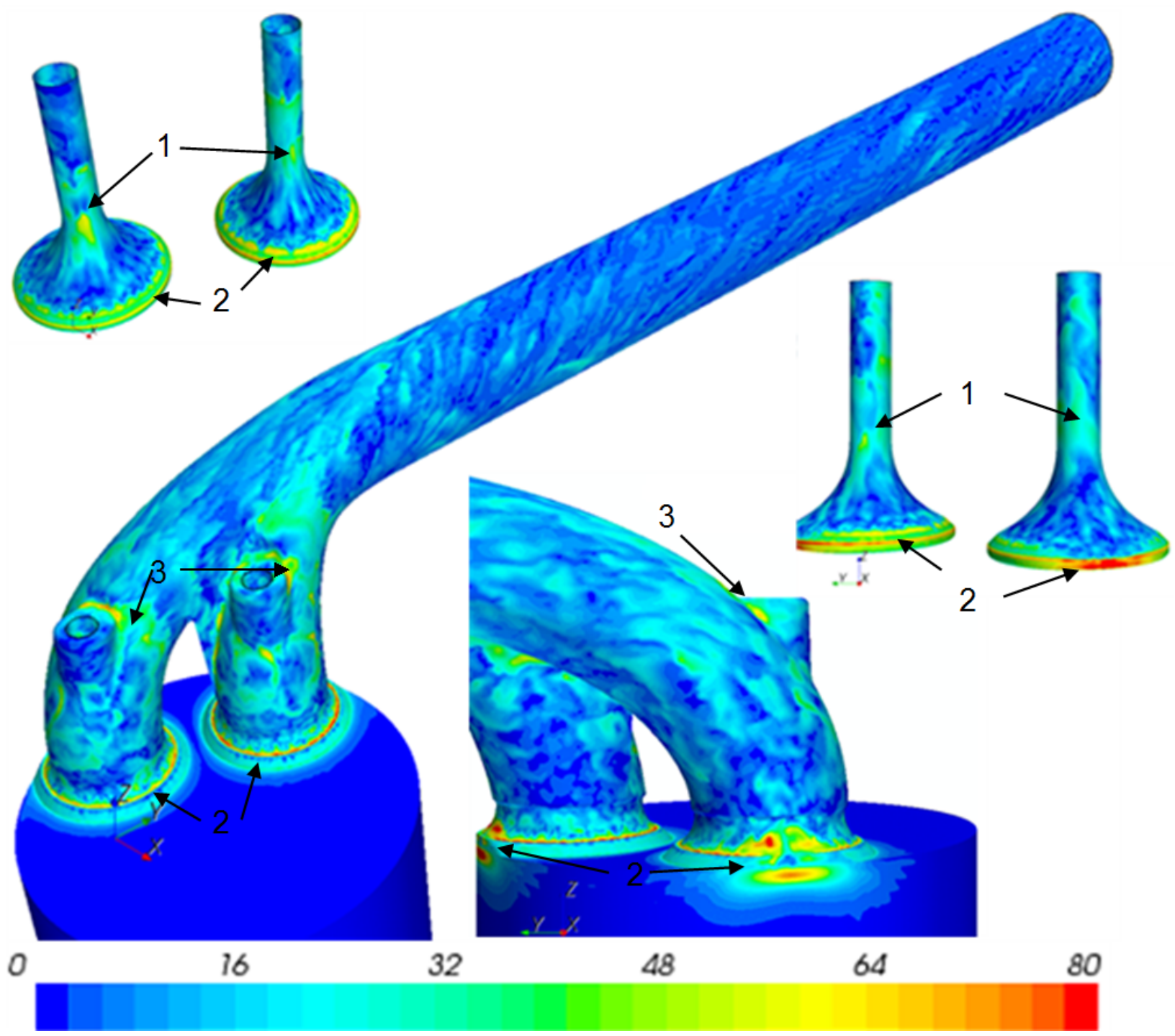

Fig. 12. The instantaneous wall shear stress magnitude $(\mathrm{Pa})$ distribution for case $\mathrm{C} 5$ is shown. 
Table 1. A tabulated overview of the computed cases. The grids 1, 2, and 3 are designed with an isotropic hexahedral core cell size of 1 , 0.8 , and $0.6 \mathrm{~mm}$, respectively.

\begin{tabular}{cccc}
\hline \hline Case & Valve lift $(\mathrm{mm})$ & Inlet mass flow $(\mathrm{kg} / \mathrm{s})$ & Grid \\
\hline $\mathrm{C} 1$ & 4 & 0.09 & 1 \\
$\mathrm{C} 2$ & 4 & 0.09 & 2 \\
$\mathrm{C} 3$ & 4 & 0.09 & 3 \\
$\mathrm{C} 4$ & 3 & 0.0664 & 3 \\
$\mathrm{C} 5$ & 4 & 0.0873 & 3 \\
$\mathrm{C} 6$ & 5 & 0.1 & 3 \\
\hline \hline
\end{tabular}

Table 2. The total pressure loss $(k P a)$ measured experimentally is compared to the results from the URANS and the LES simulations.

\begin{tabular}{cccc}
\hline \hline Valve lift & URANS & LES & Experiments \\
\hline $3 \mathrm{~mm}$ & 5.4 & 5.0 & 4.9 \\
$4 \mathrm{~mm}$ & 5.4 & 4.9 & 4.9 \\
$5 \mathrm{~mm}$ & 5.3 & 4.8 & 4.9 \\
\hline \hline
\end{tabular}


Table 3. For the $5 \mathrm{~mm}$ valve lift cases of the two data sets obtained with LES and URANS, the area weighted average of the total pressure $(k P A)$ and the total pressure loss $(k P a)$ is tabulated for and respectively between different cut planes, which are indicated in Fig. 6.

\begin{tabular}{|c|c|c|c|c|c|c|}
\hline \multirow[b]{2}{*}{ Plane } & \multicolumn{3}{|c|}{ LES } & \multicolumn{3}{|c|}{ URANS } \\
\hline & $P_{t o t}$ & $\Delta P_{t o t}$ & $\%$ & $P_{t o t}$ & $\Delta P_{t o t}$ & $\%$ \\
\hline 0 & 108.6 & - & - & 108.9 & - & - \\
\hline 1 & 107.3 & 1.3 & 27.1 & 105.7 & 3.2 & 60.4 \\
\hline 2 & 105.0 & 2.3 & 47.9 & 104.8 & 0.9 & 17.0 \\
\hline 3 & 104.5 & 0.5 & 10.4 & 104.6 & 0.2 & 3.8 \\
\hline 4 & 104.0 & 0.5 & 10.4 & 104.2 & 0.4 & 7.4 \\
\hline 5 & 103.9 & 0.1 & 2.1 & 103.9 & 0.3 & 5.7 \\
\hline 6 & 103.8 & 0.1 & 2.1 & 103.6 & 0.3 & 5.7 \\
\hline
\end{tabular}

